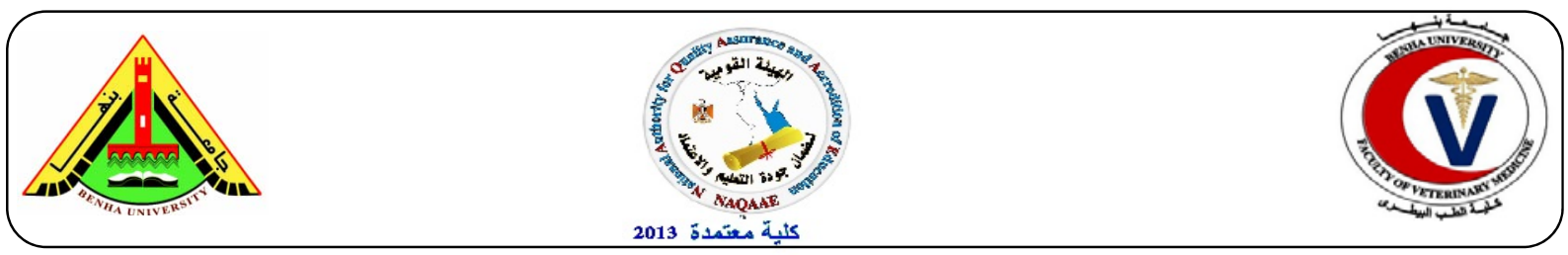

\title{
Development of Simple Multiplex Real-Time PCR Assays for Some Probiotics Detection.
}

\author{
${ }^{1}$ Yakout A. El-Senosi, ${ }^{2}$ Abdelbary M. prince, ${ }^{2}$ Adel Fathey Tohamy Afify, ${ }^{1}$ Hend“Ahmed El-Refaey \\ "Atwa. \\ ${ }^{1}$ Department of Biochemistry, Faculty of Veterinary Medicine, Benha University, Egypt. \\ ${ }^{2}$ Department of Biochemistry, Faculty of Veterinary Medicine, Cairo University, Egypt.
}

\begin{abstract}
A B S T R A C T
The main objective of this study to investigate the development of simple multiplex real time for some probiotics detection. The probiotics defined as Live microorganisms when administered in adequate amounts confer a health benefit on the host. Most commonly used probiotics are Lactic acid bacteria ( $L A B)$ and bifido bacteria. There are other examples of species used as probiotics (certain yeasts and bacilli). Probiotic supplements are popular now a day. From the beginning of 2000, research on them has increased remarkably. The beneficial effects of probiotics are now day's widely studied in treatment of many prevailing diseases. In this study, we present the development of molecular methods. Conventional multiplex PCR and SYBR Green based real time PCR assays were performed using genus and species specific primers.
\end{abstract}

Key words: Probiotics, Lactic acid Bacteria ( $L A B)$, Bifido bacteria, Conventional multiplex PCR, SYBR Green Real time PCR.

\section{INTRODUCTION:}

Probiotics denotes "for life" and refers to microorganisms which have positive effects on human health. Micro-organisms presiding in food, especially lactic acid bacteria were believed to have beneficial health (Lorena Carro, et al., 2017). Probiotic bacteria are mainly lactic acid bacteria, lactobacilli in particular, but they also include other bacteria, such as bifidobacteria and propioni bacteria (Sanja Kolac`ek, et al., 2017). D Dairy yogurt is produced using a culture of Lactobacillus delbrueckii subsp. bulgaricus and Streptococcus thermophilus bacteria. In addition, other lactobacilli and bifidobacteria are also sometimes added during or after culturing yogurt. (Sara Tomas, 2017). The polymerase chain reaction (PCR) is now a common and often indispensable technique used in medical and biological research labs for a variety of applications. These include DNA cloning for sequencing, DNA-based phylogeny, or functional analysis of genes; the diagnosis of hereditary diseases; the identification of genetic fingerprints (used in forensic sciences and DNA paternity testing); and the detection and diagnosis of infectious diseases.(Kary Mullis,1993). Aim of the study to alter ate of the development of simple multiplex real time for some probiotics detection.

\section{MATERIALS AND METHODS:}

\subsection{Lactobacilli strains and isolates:}

In this study, we isolated strains from commercially available yoghurts mentioned in Table 1. (Danner et al. 2003), LBS agar. These methods included morphology screening and biochemical tests (API 50 CHL; bioMerieux, Nurtingen, Germany). In addition, species identification was carried out by MALDI-TOF MS using a Microflex LT instrument, FlexControl 3.0 software and the BioTyper 3.0 database (Bruker Corporation, Billerica, MA, USA) (Murugaiyan et al. 2012).

\subsection{DNA extraction from pure lactobacilli cultures:}

We used a DNA extraction method modified from Walter et al. (2000).

\subsection{DNA extraction from yoghurt:}

DNA was isolated from yoghurt using the method described in Lick et al. (1996) 


\subsection{Quantitative Real-time PCR:}

PCR is a powerful technique to detect and amplify fragments of DNA, and soon after its discovery in 1983 by Kari Mullis (Mullis, et al., 1986)., (VanGuilder, et al., 2008), (Nolan, et al., 2006).

\subsection{Statistical analysis:}

The obtained data were analyzed represented using the statistical package for social science (SPSS, 13.0 software, 2009), for obtaining mean and standard deviation and error. The data were analyzed using one-way ANOVA to determine the statistical significance of differences among groups. Duncan's test was used for making a multiple comparison among the groups for testing the inter-grouping.

\section{RESULTS}

\subsection{Species-specific amplification of lactobacilli DNA:}

Initially, different target regions had been tested for the species-specific detection of members of the genus Lactobacillus. We were able to establish species-specific primer pairs to detect different strains of the species Lactobacillus acidophilus, Lact. brevis, Lact. delbrueckii subsp. bulgaricus, Lact. helveticus and Lact. reuteri using annealing temperatures shown in Table 2. Using Lactobacillus DNA from pure cultures and product isolates from yoghurt showed CT values ranging from 14 to 28 threshold cycles. Unspecific signals started from the 32nd cycle on; however, they were easily distinguishable by melting curve analysis. The latter unspecific amplification signals were induced by small artefacts due to primer dimers, which were proven by agarose gel electrophoresis. Conclusively, using CT value and the melting curve analysis allowed a distinct species-specific identification of all lactobacilli species which are detectable. The specificity of the assay was tested using product isolates from yoghurt of Lact. delbrueckii subsp. bulgaricus and Lact. acidophilus. Both species isolated from different products were assigned correctly to the respective Lactobacillus species using our assay.

\subsection{Detection limit, identification and quantification of Lactobacillus isolates from yoghurt}

To correlate amplification curves and CT values with colony -forming units and the actual number of bacteria present in one sample, we performed serial dilution experiments as described in the Methods section. This enables assumptions concerning the amount of bacteria present in a sample based on the CT value obtained by realtime PCR. By this the detection limit of the assay was observed between 104 and $106 \mathrm{CFU} \mathrm{ml} 1$ of all lactobacilli tested (Fig. 1, Table 2). Two commercially available yoghurts (Activia; Danone and Proviact Bifidus pur ; HMI GmbH) were used to verify whether an identification and quantification is possible using our developed assay. Therefore, DNA was directly isolated from these products without prior cultivation. We were able to identify Lact. Delbrueckii subsp. bulgaricus correctly by real-time PCR assay in the Activia yoghurt sample, which is used as a starting culture running the fermentation process. Additional melting curve analyses (Fig. 2) revealed nearly similar melting curves of the 'unknown' yoghurt DNA sample and the serial dilutions of reference strain DNA from Lact. delbrueckii subsp. bulgaricus. These serial dilutions enabled us to estimate the number of Lact. Delbrueckii subsp. bulgaricus present in yoghurt samples based on the correlating amplification curve of the DNA mixture we extracted from Activia yoghurt to range between 107 and $108 \mathrm{CFU} / \mathrm{ml}$ yoghurt. We were able to detect the strain Lact. delbrueckii subsp. bulgaricus within the DNA mixture extracted from Activia, which was mentioned on the product's package. In Proviact Bifidus pur (HMI $\mathrm{GmbH}$ ), we were able to detect Lact. acidophilus using species-specific primer pairs, although this species was not mentioned in the nutritional facts.

Table 1 Field isolates obtained from yoghurt in this study:

\begin{tabular}{ll}
\hline Species & Origin \\
\hline Lactobacillus delbrueckii subsp. bulgaricus) & Nestle youghurt \\
& Elmaraei yoghurt \\
Lactobacillus acidophilus & Activia yoghurt \\
& Lactel yoghurt \\
& Juhayna yoghurt \\
& Danone yoghurt \\
\hline
\end{tabular}


Table 2: Primer sequences for internal control genes:

\begin{tabular}{|c|c|c|c|c|c|c|c|c|c|c|}
\hline \multirow[t]{2}{*}{ IDL04F } & AGG GTG AAG TCG TAA & 160401B006H0952/126 & 24 & Desalted & 284.0 & 7.451 & 65 & 5.0 & 17.5 & 175 \\
\hline & CAAGTAGCC & & & & & & & & & \\
\hline \multirow[t]{2}{*}{ IDL11F } & TGG & 160401B006A1053/126 & 24 & Desalted & 262.0 & 7.440 & 67 & 6.8 & 25.8 & 258 \\
\hline & TCGGCAGAGTAACTGTTGTCG & & & & & & & & & \\
\hline \multirow[t]{2}{*}{ IDL22R } & AAC TAT CGC TTA CGC TAC & 160401B028G0154/126 & 26 & Desalted & 268.0 & 7.841 & 66 & 4.5 & 16.8 & 168 \\
\hline & CAC TTTGC & & & & & & & & & \\
\hline \multirow[t]{2}{*}{ IDL31F } & CTG TGC TAC ACCTAGAGA & 160401B006B1055/126 & 25 & Desalted & 279.0 & 7.722 & 67 & 6.4 & 22.9 & 229 \\
\hline & TAG GTG G & & & & & & & & & \\
\hline \multirow[t]{2}{*}{ IDL42R } & ATT TCA AGT TGA & 160401B006C1056/126 & 23 & Desalted & 234.0 & 6.956 & 59 & 5.0 & 21.3 & 213 \\
\hline & GTCTCTCTC TC & & & & & & & & & \\
\hline \multirow[t]{2}{*}{ IDL52F } & ACC TGA TTG ACGATG GAT & 160401B006D1057/126 & 25 & Desalted & 278.0 & 7.666 & 66 & 5.5 & 19.6 & 196 \\
\hline & CAC CAG T & & & & & & & & & \\
\hline \multirow[t]{2}{*}{ IDL62R } & CTA GTGGTAACAGTT GAT & 160401B028H0158/126 & 27 & Desalted & 313.0 & 8.332 & 64 & 4.9 & 15.5 & 155 \\
\hline & TAA AAC TGC & & & & & & & & & \\
\hline \multirow[t]{2}{*}{ IDL73R } & GCC AAC AAG CTA TGT & 160401B006E1059/126 & 25 & Desalted & 264.0 & 7.633 & 67 & 5.3 & 20.1 & 201 \\
\hline & GTTCGC TTGC & & & & & & & & & \\
\hline
\end{tabular}




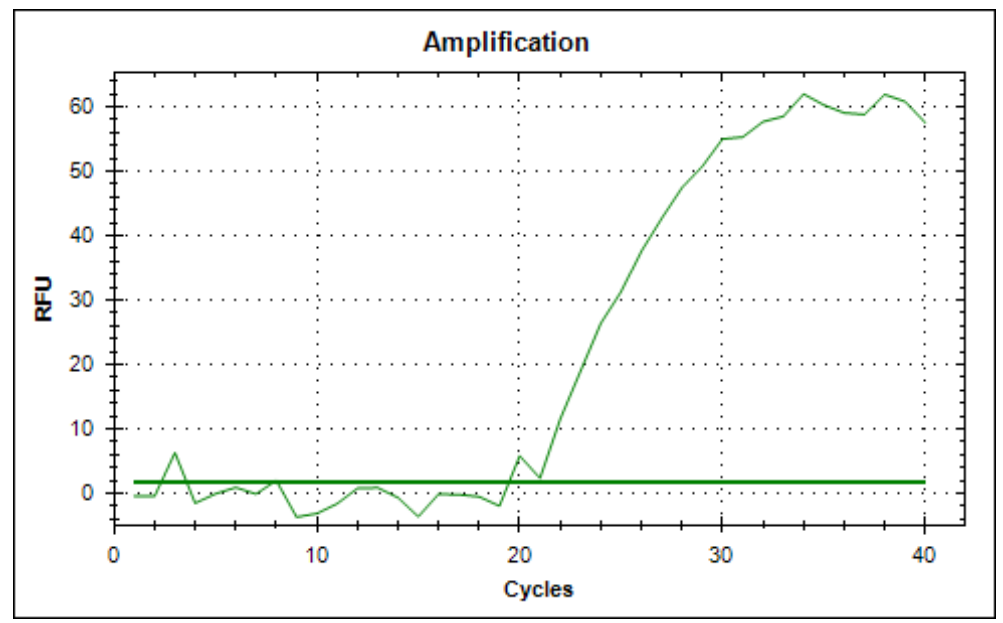

Figure 1. Quantification of Lactobacillus delbrueckii subsp. bulgaricus in DNA mixture extracted from Activia using real-time PCR.

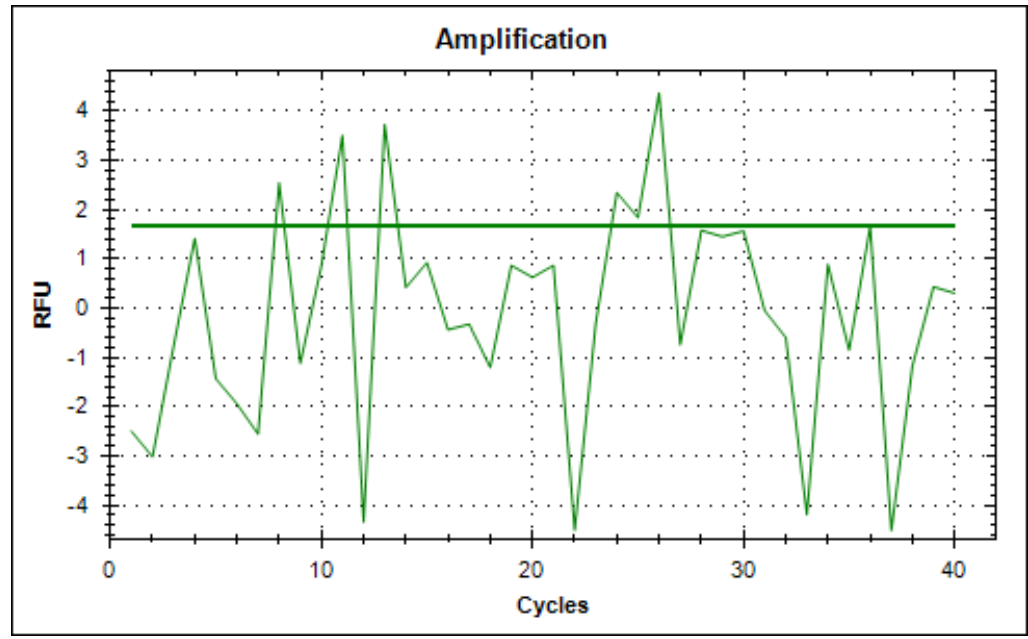

Figure 2: Amplification curves of DNA isolate from yoghurt and negative controls containing Lact. delbrueckii subsp. bulgaricus primers.

\section{DISCUSSION:}

Herein, we describe a real-time PCR approach for the rapid detection and quantification of important probiotic lactobacilli using DNA directly isolated from yoghurt. Our screening for an appropriate target region resulted in targeting the heat shock protein region (hsp60). Other putative target regions including the 23S-5S rRNA (intergenic spacer region) and rpoA (Berthier and Ehrlich 1998; Kwon et al. 2004; Cebeci and Gurakan 2011) showed no sufficient species specification due to false-positive amplification signals of negative controls. Primers targeting those regions were therefore excluded from further development, and we concentrated our work on the heat shock protein region hsp60. Using the BLAST algorithm, the sequences of the used primers were confirmed to solely target the genome of lactobacilli and no other genes were found showing a comparable DNA sequence. This conserved region appears to be a suitable target for the identification of lactobacilli as previous studies obtained similar results for other species such as Staphylococcus aureus and Staphylococcus epidermidis or Legionella pneumophila and species of the genus Bifidobacterium (Goh et al. 1996; Blaiotta et al. 2008; Karapetsas et al. 2010; Sun et al. 2010; Junick and Blaut 2012; Park et al. 2012; Yu et al. 2012; de Boer et al. 2013). One of the key advantages of this assay is its rapidity as it allows species-specific identification of lactobacilli strains within $7 \mathrm{~h}$ without any prior cultivation. In contrast, culture-dependent techniques such as API 50 CHL stripes, 
conventional colony PCR plus sequencing or using MALDI-TOF MS need up to $96 \mathrm{~h}$ to identify the isolated bacteria to species level. However, additionally utilized methods confirm the specificity of the described quantitative real-time PCR assay. The sensitivity of the assay is sufficient as the detection limit measured $\left(10^{5} \mathrm{CFU} / \mathrm{ml}\right)$ is adequate to quantify strains of starting cultures and advertised species fortified in yoghurt in a range of $10^{6}-10^{8} \mathrm{CFU} / \mathrm{ml}$ to exert probiotic activity (Robinson 1987; Shah 2000).

To quantify probiotic bacteria in food, a vigorous usage of ultrasonic sound system had a positive effect on the assays' sensitivity. Ultrasonic sound usage for a duration of $30 \mathrm{~s}$ on the cells of Lactobacillus spp. strains in overnight cultures, serial dilutions and diluted yoghurt samples disrupts the cells spreading the DNA throughout the sample, thus leading to earlier CT values and smaller standard derivations. Besides interpretation of the amplification curves, melting curve analysis presents an important parallel approach to assure that the amplicon generated is species-specific and not an artifact due to primer dimers. However, melting curve analysis of pure lactobacilli DNA and DNA mixtures isolated from yoghurt revealed differences in the melting temperature of the amplicons up to $1{ }^{\circ} \mathrm{C}$ (Fig. 2). Nevertheless, by sequencing the amplicons obtained from yoghurt samples, we were able to show that the sequences were specific for the targeted strain in the yoghurt thus no false positives (Fig. S1). A possible reason for the differences in the melting temperature might be the formation of heteroduplex complexes caused by using mixtures of DNA in comparison with the homoduplexes in pure DNA (Zhou et al. 2004; Maeder et al. 2008).

\section{CONCLUSION:}

So we concluded that, this newly developed real-time PCR assay is detecting Lact. acidophilus, Lact. brevis, Lact. Delbrueckii subsp. bulgaricus, Lact. helveticus and Lact. Reuteri with high specificity, sensitivity and without any false positive signals from other lactobacilli from pure cultures or DNA mixtures extracted from yoghurt. In contrast to other identification tools (physiological testing, morphology), the real-time PCR system enables the user to identify and quantify detectable strains of the genus Lactobacillus within a day including the DNA extraction from yoghurt. Therefore, this real-time PCR assay might be a useful method for the detection of Lactobacillus ssp. strains in food for regulation and quality management purposes.

\section{REFERENCES:}

Berthier Françoise, Stanislav Dusko Ehrlich. 1998. Rapid species identification within two groups of closely related lactobacilli using PCR primers that target the $16 \mathrm{~S} / 23 \mathrm{~S}$ rRNA spacer region. FEMS Microbiology Letters; 161, (1), 1 April: 97-106.

Blaiotta Giuseppe,Vincenzina Fusco, Danilo Ercolini, Maria Aponte, Olimpia Pepe and Francesco Villani. 2008. Lactobacillus Strain Diversity Based on Partial hsp60 Gene Sequences and Design of PCRRestriction Fragment Length Polymorphism Assays for Species Identification and Differentiation. Appl. Environ. Microbiol. January ,. $74 ; 1: 208-215$

Cebeci Aysun, G., Candan Güra . 2011. Comparative typing of L. delbrueckii subsp. bulgaricus strains using multilocus sequence typing and RAPD-PCR. European Food Research and Technology. September, 233;3: 377-385

Danner. H ,.Holzer. M., Mayrhuber . E., Braun, R. 2003. Acetic Acid Increases Stability of Silage under Aerobic Conditions. Department of Environmental Biotechnology, Institute for Agrobiotechnology IFA-Tulln, Konrad Lorenz January. $69 ; 1$ :562-567.

de Boer Onno J, Felipe De Sousa E Melo, Xin Wang, Marnix Jansen, Evelyn Fessler, Anne Trinh, Laura P M H de Rooij, Joan H de Jong, Ronald van eersum, Maarten F Bijlsma, Hans Rodermond, Maartje van der Heijden, Carel J M van Noesel, Jurriaan B Tuynman, Evelien Dekker,Florian Markowetz, Jan Paul Medema, Louis Vermeulen. 2013. Poor-prognosis colon cancer is defined by a molecularly distinct subtype and develops from serrated precursor lesions. Nature Medicine;19:614618.

Goh, S H., Potter, S., Wood, J O ., Hemmingsen, S M ., Reynolds, R P , Chow, A W. 1996. HSP60 gene sequences as universal targets for microbial species identification: studies with coagulasenegative staphylococci. J. Clin. Microbiol. April ; 34 ; 4:818-823.

Junick Jana, Michael Blaut. 2012. Quantification of Human Fecal Bifidobacterium Species by Use of Quantitative Real-Time PCR Analysis Targeting the groEL Gene . Appl. Environ. Microbiol. April ; 78; 8: 26132622. 
Karapetsas, A., Vavoulidis, E., Galanis, A., Sandaltzopoulos, R., Kourkoutas Y., 2010 - Rapid Detection and Identification of Probiotic Lactobacillus casei ATCC 393 by Multiplex PCR. J Mol Microbiol Biotechnol.18:156-161

Kary Mullis. 1993. the Polymerase Chain Reaction .Nobel Lecture, December 8 . 64-65.

Kwon, Li., Yougen ., Tseng ., Yolanda, D., Sang, Y., d'Espaux, Leo., Bunch, J Scott. 2004. Controlled assembly of dendrimer-like DNA. Nature Materials; London 3.1 ;Jan: 38-42.

Lick, S., Keller, M., Bockelmann, W., Heller, K.J. 1996. Optimized DNA extraction method for starter cultures from yoghurt. Milchwissenschaft .51: 183-186.

Lorena Carro , Imen Nouioui . 2017. Taxonomy and systematics of plant probiotic bacteria in the genomic. Microbiology, 3;3: 383-412.

Maeder Morgan, L., Stacey ThibodeauBegannyAnnaOsiak, David A., Wright ,Reshma Anthony ,Magdalena Eichtinger, TaoJiang, Jonathan E., Foley, Ronnie, J., Winfrey, Jeffrey, A., Townsend, Erica Unger-Wallace, Jeffry D.Sander ,Felix Müller -Lerch, FengliFu, Joseph Pearlberg, CarlGöbel, Justin P. Dassie, Shondra M. Pruett-Miller, J. Keith Joung . 2008. Rapid "Open-Source" Engineering of Customized Zinc-Finger Nucleases for Highly Efficient Gene Modification . 31; 2: 25 July: 294-301.

Mullis, k., Faloona, F., Scharf, S., Saiki, R., Horn, G., Erlich, H. 1986. Specific Enzymatic Amplification of DNA in Vitro: The Polymerase Chain Reaction. Cold Spring Harb Symp Quant Biol. 51: 263-273

Murugaiyan Gopal, Oleg Butovsky, Shafiuddin Siddiqui, Galina Gabriely, Amanda J. Lanser, Ben Dake, Camille E. Doykan, Pauline M. Wu, Reddy R. Gali, Lakshmanan K. Iyer, Robert Lawson, James Berry, Anna M. Krichevsky, Merit E. Cudkowicz, Howard L. Weiner. 2012. Modulating inflammatory monocytes with a unique microRNA gene signature ameliorates murine ALSJ .Clin Invest. 4; 122;9: 3063 3087.

Nolan, Tania, Hands, Rebecca, E., Bustin, Stephen, A. 2006. Quantification of mRNA using real-time RT-PCR. Nature Protocols; London 1.3 ;Aug: 1559-82.

Park Sang-Cheol ,Ok-Sun Kim, Yong-Joon Cho, Kihyun Lee, Seok-Hwan Yoon, Mincheol Kim, Hyunsoo Na, Yoon Seong Jeon, Jae-Hak Lee, Hana
Yi, Sungho Won, Jongsik Chun. 2012. Introducing EzTaxon-e: a prokaryotic $16 \mathrm{~S}$ rRNA gene sequence database with phylotypes that represent uncultured species. International Journal of Systematic and Evolutionary Microbiology. 62: 716721.

Robinson Campbell, W., MilanPopović . 1987.The specific interfacial area in externalcirculation-loop airlifts and a bubble column-II. Carboxymethyl cellulose/ sulphite solution. Chemical Engineering Science; 42;12 : 2825-2832.

Sanja Kolac`ek, Iva Hojsak, Roberto Berni Canani, Alfredo Guarino, Flavia Indrio, Rok Orel, Bruno Pot, Raanan Shamir, Hania Szajewska, Yvan Vandenplas, Johannes van Goudoever, AQ2 Zvi Weizman . 2017. Commercial Probiotic Products. CE: R.R.; JPGN; 17; 207: 209.

Sara Thomas. 2017. Activia - probiotic. https://www.activia.us.com .July.2:84-99.

Shah, N.P. 2000. Probiotic Bacteria: Selective Enumeration and Survival in Dairy Foods. Journal of Dairy Science.83; 4, April :894907.

Stastical Analysis system (S.A.S). (2002): Version 9.00 .S.A.S Institute Inc., cary, NC,USA.

Sun Liang, Andrea Splendiani, Yuanbo Zhang, Tianshu Li , Jonghwan Kim , Chi-Yung Chim, Giulia Galli and Feng Wang. 2010. Emerging Photoluminescence in Monolayer $\mathrm{MoS}_{2}$. Nano Lett., 10 ;4: 1271-1275.

VanGuilder Heather, D., Kent E Vrana, Willard M Freeman. 2008. Twenty-five years of quantitative PCR for gene expression analysis. Biotechniques .4;44:5:619.

Walter, F., Wu , Xiaohua, Ranganathan, Velvizhi, Weisman, David, S., Heine. 2000. ATM phosphorylation of Nijmegen breakage syndrome protein is required in a DNA damage response. Nature; London .405.6785 May 25: 477-82.

$\mathrm{Yu}$ Dong, Geoffrey Hinton, Li Deng_George E. Dahl, Abdel-rahman Mohamed, Navdeep Jaitly, Andrew Senior, Vincent Vanhou. 2012. Deep Neural Networks for Acoustic Modeling in Speech Recognition: The Shared Views of Four Research Groups. IEEE Signal Processing Magazine. 29;6, Nov:s: 82 - 97.

Zhou Wang, Bovik, A. C., Sheikh, H.R., Simoncelli, E.P. 2004. Image quality assessment: from error visibility to structural similarity.13;4, April:(s): $600-612$. 